\title{
Reliability of the Longitudinal Welds of LHC Main Dipoles
}

\author{
Paolo Fessia, Cristiano Lanza, and Stefano Sgobba
}

\begin{abstract}
The LHC main dipoles are assembled relying heavily on welding technology. In particular, two $15 \mathrm{~m}$ long longitudinal MAG welds along the shrinking cylinder, manufactured from AISI 316 LN stainless steel plates, close the cold mass and are submitted to tensile stress. The welds have to feature a design stress of $350 \mathrm{MPa}$ at $1.8 \mathrm{~K}$ and to guarantee the leak tightness to superfluid helium. Starting from available experimental data, the reliability of the cold mass welds in terms of fracture mechanics is studied under different hypotheses (i.e., presence of surface or embedded flaws). The analysis has been carried out using a wide range of different approaches, such as Leak before break, LCF (Low Cycle Fatigue), LEFM (Linear Elastic Fracture Mechanics) and established standards (WES 2805); also different approaches of propagation under fatigue are compared. The results of this study are meant to prove the robustness and reliability of the design at the working temperature of the magnets.
\end{abstract}

Index Terms-Crack, fatigue, weld, 316LN.

\section{INTRODUCTION}

W ELDING technology is widely used for the assembly of the 1248 dipoles. In particular this applies to the two $15 \mathrm{~m}$ long longitudinal welds (as indicated in the Fig. 1) on the shrinking cylinder, manufactured from AISI 316 LN stainless steel plates which close the cold mass.

These welds are performed bringing together the chamfered edges under a large press and are made by 4 runs: after the first STT (Surface Tension Transfer ${ }^{1}$ ) root pass, three MAG filler passes complete the weld (see Fig. 2).

The design methods considered to analyze the weld reliability are the Barsom-Rolfe approach used in [1] and in [2] and the WES 2805: "Method of Assessment for Flaws in Fusion welded joints with respect to Brittle Fracture and fatigue crack growth" [3] used in ATLAS experiment at CERN [4].

Moreover some improved formulas (reported in Appendix) and some safety factors have been implemented in order to take into account possible deviations from nominal conditions have been implemented.

The WES standard has been identified as the most conservative approach and has been used to characterize the reliability with respect to possible flaws of the longitudinal weld main dipole.

Manuscript received October 21, 2003.

P. Fessia and C. Lanza are with the CERN (European Organization for Nuclear Research) Accelerator Technology Division, 1211 Geneva 23, Switzerland (e-mail: paolo.fessia@cern.ch; cristiano.lanza@cern.ch).

S. Sgobba is with the CERN (European Organization for Nuclear Research) Engineering Support and Technologies Division, 1211 Geneva 23, Switzerland (e-mail: stefano.sgobba@cern.ch).

Digital Object Identifier 10.1109/TASC.2004.830514

${ }^{1} \mathrm{STT}$ is a trademark of Lincoln Electric.

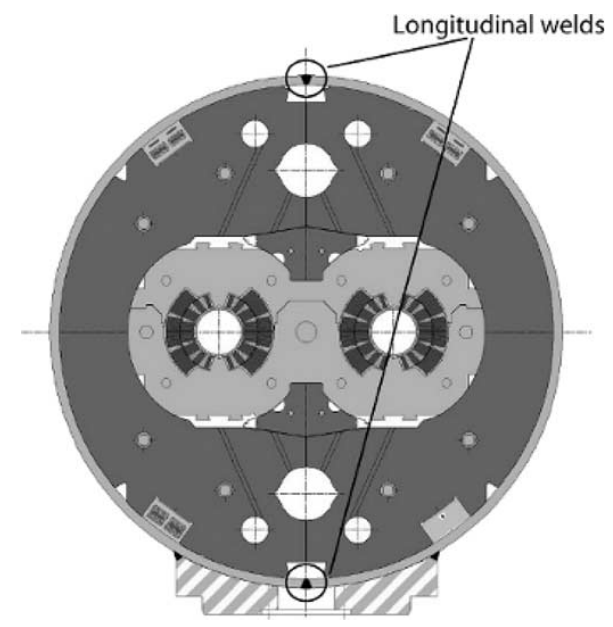

Fig. 1. LHC main dipole cross section.

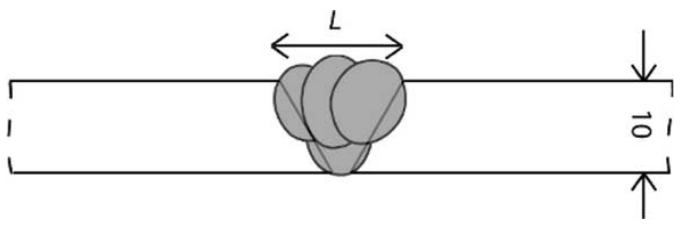

Fig. 2. Longitudinal weld description (thickness is $10 \mathrm{~mm}, \mathrm{~L}$ is $\sim 15 \mathrm{~mm}$ ).

\section{COMPUTATION}

The linear defects observed in the welds of the LHC main dipole can be of various types: general flaws, lack of fusion or lack of penetration. This analysis addresses two types of defects: embedded and surface flaws. Inter-run lack of fusion can be classified as embedded flaw. Lack of penetration or lack of fusion at the weld root can be classified as surface flaws.

For each case, the critical dimensions of a crack were calculated and the influence of fatigue cycles was considered.

We treated a surface flaw as a semi-elliptical surface crack with depth of $a$ and length of $2 c$ and an embedded flaw as elliptical embedded crack with depth of $2 a$ and length of $2 c$ (see Fig. 3). Due to the loading conditions the local displacements, around the weld, are symmetric respect to the center plane of weld itself. Therefore here the Mode I [5] can be assumed as the loading mode to define the methods for the stress analysis for cracks.

Due to practical constraints (timeframe, cost, thickness of the plate) it is necessary to evaluate indirectly the critical stressintensity factor $K_{I c}$ from other known or measured parameters. Such correlations (Lower bound, Roberts and Newton [5]; Upper shelf Barsom and Rolfe [5]; the formula developed by Tobler [6]) are widely used and have been used for application 

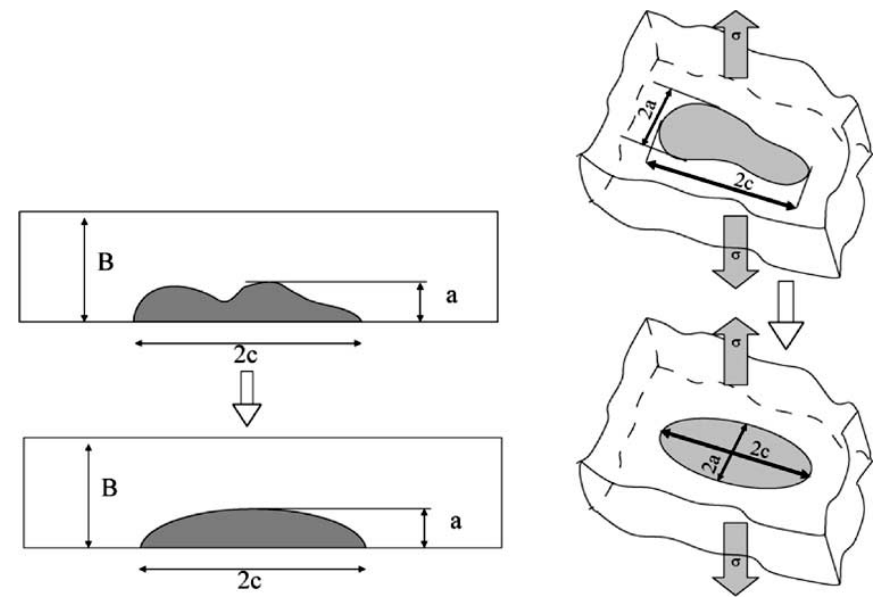

Fig. 3. Surface and embedded crack approximations.

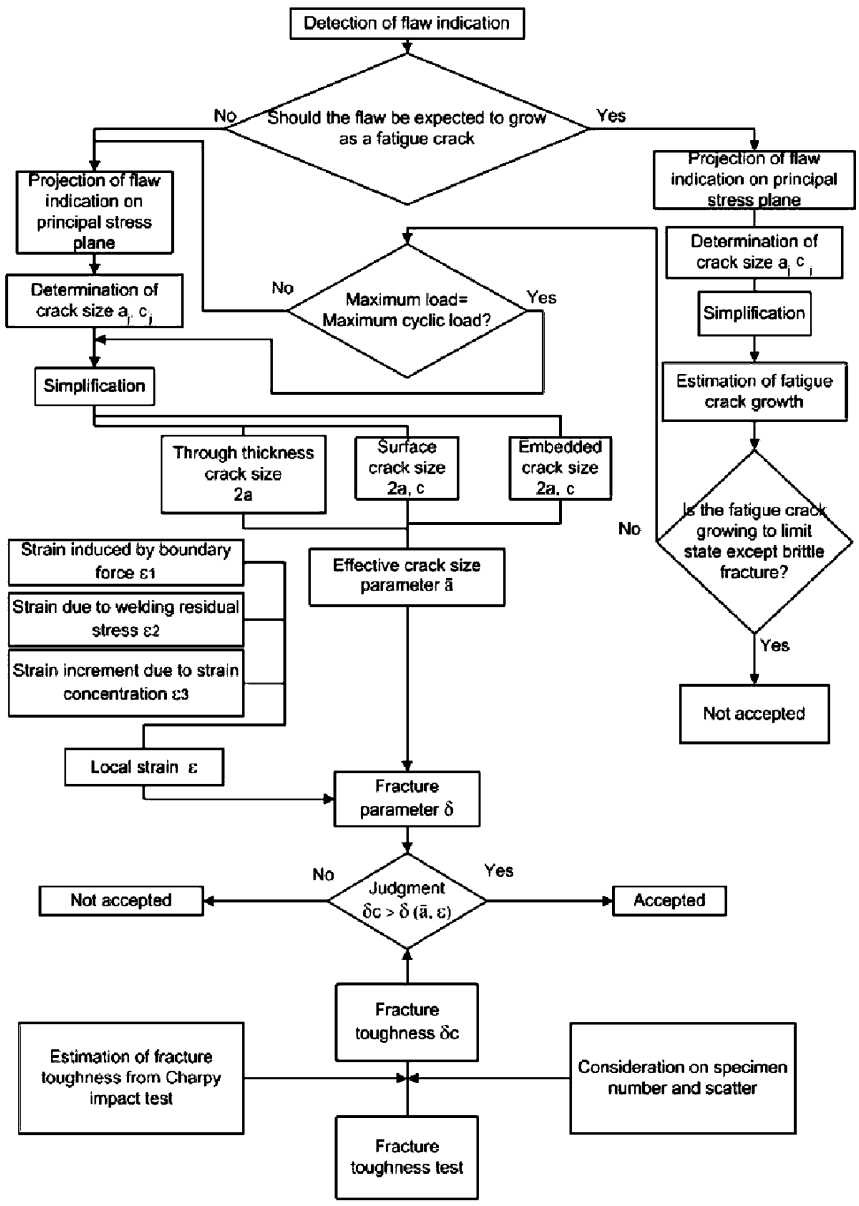

Fig. 4. WES procedure for flaw assessment.

in the sector of high field superconducting magnets. With the previous correlations and using the available experimental data (CVN tests on weld metal and on the heat affected zone) a conservative $K_{I c}$ value of $100 \mathrm{MPa} \sqrt{ } \mathrm{m}$ has been deduced and fixed for the rest of this work.

For the assessment for flaws in the dipole longitudinal weld the Japanese Standard WES 2805 has been used (see Fig. 4).

The design stress value for the longitudinal weld at room temperature is $150 \mathrm{MPa}$. While at the working temperature of $1.8 \mathrm{~K}$
TABLE I

PARAMETERS FOR THE PARIS LAW

\begin{tabular}{lccc}
\hline \hline & Temperature & $C$ & $m$ \\
\hline WES & RT & $2.60 \times 10^{-11}$ & 2.75 \\
$316 \mathrm{LN}$ & $4 \mathrm{~K}$ & $2 \times 10^{-12}$ & 3.5 \\
\hline
\end{tabular}

(Where units of crack length, stress and stress-intensity factor are [m], [MPa], $[\mathrm{MPa} \sqrt{\mathrm{m}}]$, respectively)

the design value is $340 \mathrm{MPa}$, the results of a Monte Carlo simulation [7] confirm that the maximum possible value is $\sim 370$ $\mathrm{MPa}$. In order to increase the safety margin, an upper value of $400 \mathrm{MPa}$ has been used in the computations.

The dipoles have to withstand at least 25 thermal cycles between room temperature and $1.8 \mathrm{~K}$.

For the estimation of fatigue crack growth a cyclic load of $220 \mathrm{MPa}$ due to the thermal cycles has been considered. Being in the impossibility to evaluate the robustness through the real cycle evaluations, the resistance at fatigue with constant material properties at 293 and $1.8 \mathrm{~K}$ has been evaluated.

A general description of the fatigue behavior is given by the Paris law [1], [3];

$$
\frac{d l}{d N}=C\left(\Delta K^{m}-\Delta K_{t h}^{m}\right)
$$

where: $l$ is the crack size, $N$ is the number of cycles and $\Delta K_{t h}$ is the threshold of stress intensity factor range. In the literature it is possible to find different values for the parameters $C$ and $m$ : in this work the values proposed in the standard WES for room temperature and the values found specifically for the 316LN at $4 \mathrm{~K}$ have been used:

From the results shown in Figs. 7, 8, 9, and 10, it is possible to conclude that:

1) Surface cracks are much more critical than embedded, for the same thickness of material affected.

2) The cryogenic working conditions are more severe, being the number of admissible cycles equal or smaller at $4 \mathrm{~K}$ than at $293 \mathrm{~K}$.

As results from the performed computations it is possible to affirm that the effect of fatigue can be neglected because there is no remarkable crack growth over limited number of cycles that could cause brittle fracture.

In order to identify the critical dimensions of a crack, the fracture parameters have been evaluated according to the WES procedure. The effective crack size parameter $\bar{c}$ has been determined as follows [3]:

$$
\bar{c}=a F_{t}^{2}
$$

where $F_{t}$ is the shape parameter for the tensile stress and depends in particular on the flaw and weld dimensions.

Using the effective crack size $\bar{c}$ and the local strain $\varepsilon$ the fracture mechanics parameter $\delta$ can be determined as follows [4] where the $\varepsilon_{y}$ is the yield strain:

$$
\begin{aligned}
& \delta=\varepsilon_{y} \bar{c} \cdot\left(\frac{\pi}{2}\right) \cdot\left(\frac{\varepsilon}{\varepsilon_{y}}\right)^{2} \quad \frac{\varepsilon}{\varepsilon_{y}} \leq 1 \\
& \delta=\varepsilon_{y} \bar{c} \cdot\left(\frac{\pi}{8}\right) \cdot\left\lfloor 9\left(\frac{\varepsilon}{\varepsilon_{y}}\right)-5\right\rfloor \quad \frac{\varepsilon}{\varepsilon_{y}}>1 .
\end{aligned}
$$




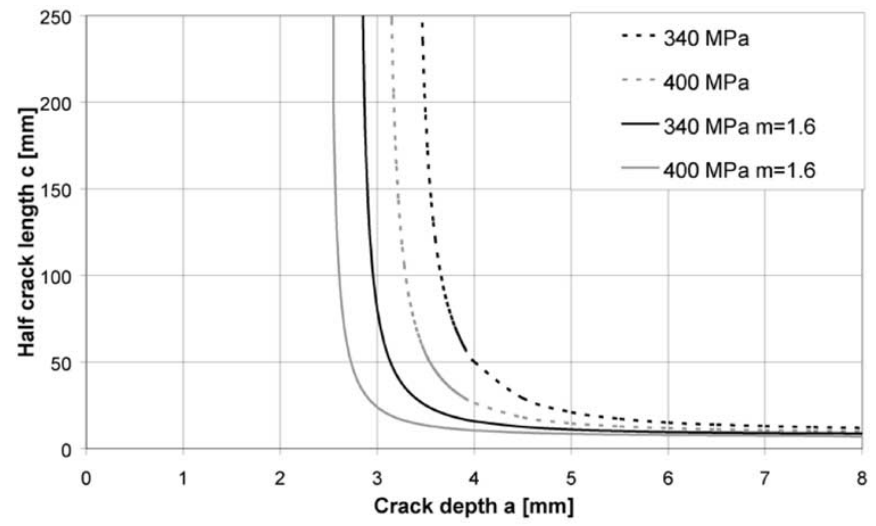

Fig. 5. Critical dimension for a surface crack; values of depth $a$ and half length $c$ as a function of the stress $\sigma$ and the constraint factor $m=1$ and $m=1.6$.

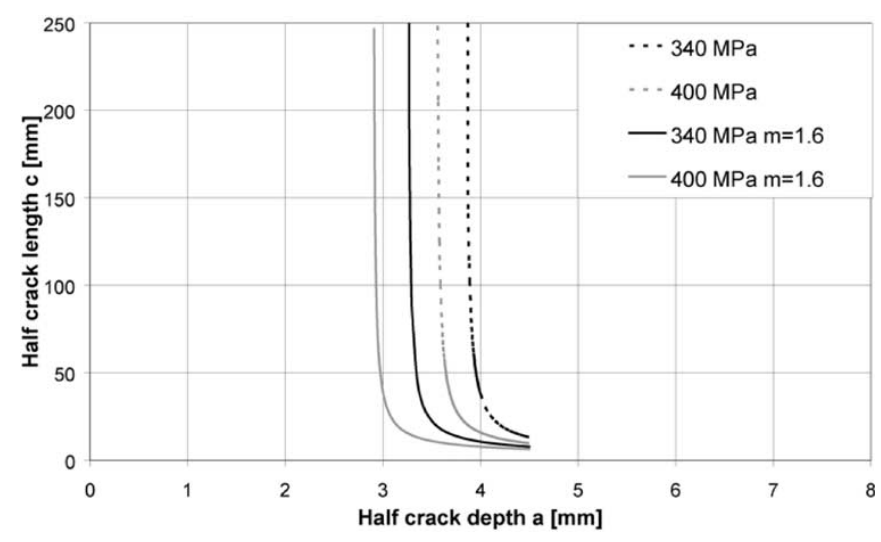

Fig. 6. Critical dimension for an embedded crack; values of half depth $a$ and half length $c$ as a function of the stress $\sigma$ and the constraint factor $m=1$ and $m=1.6$.

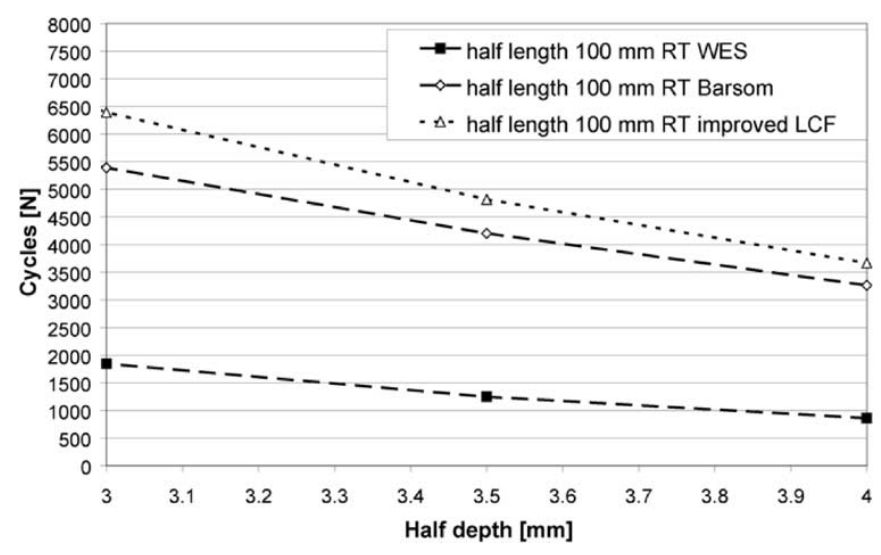

Fig. 7. Fatigue cycles for a surface crack: comparison at room temperature between improved LCF formula, Barsom and WES.

Comparing the fracture parameter $\delta$ with fracture toughness $\delta_{c}$, if $\delta_{c}>\delta$ for the crack to be assessed $\delta$ is less than $\delta_{c}$ may be judged to be acceptable; in fact the crack is critical when $\delta=\delta_{c}$.

A possible estimation for $\delta_{c}$ is [5]

$$
\delta_{c}=\frac{K_{I c}^{2}}{m E \sigma_{y s}}
$$

where the constraint factor $m$ varies from 1.2, for plane stress, to 1.6 in case of completely plane strain behavior [5] and therefore it takes into account loading conditions for thin plates.

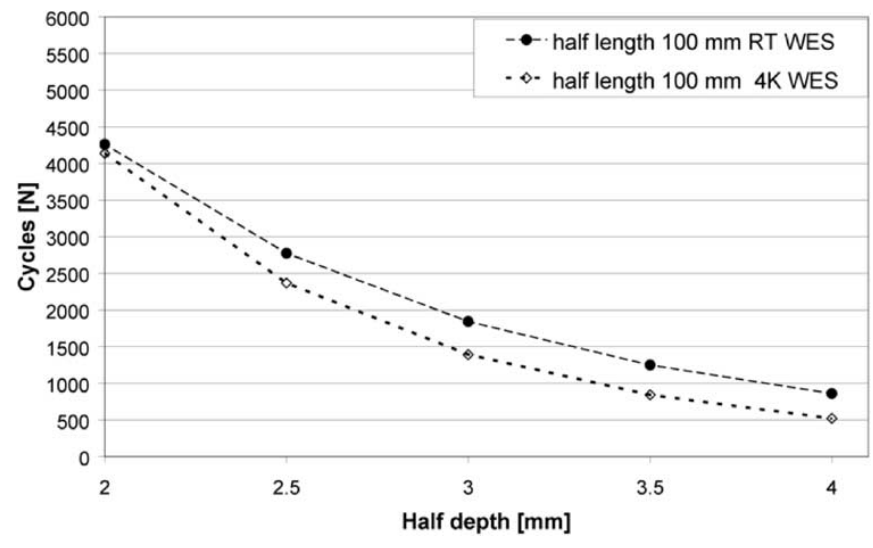

Fig. 8. Fatigue cycles for a surface crack: WES comparison RT \& $4 \mathrm{~K}$.

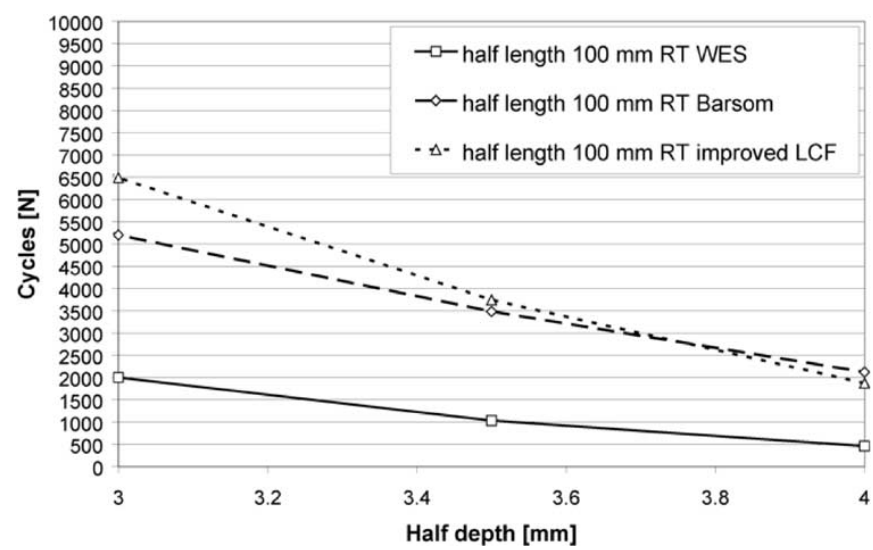

Fig. 9. Fatigue cycles for an embedded crack: comparison at room temperature between improved LCF formula, Barsom and WES.

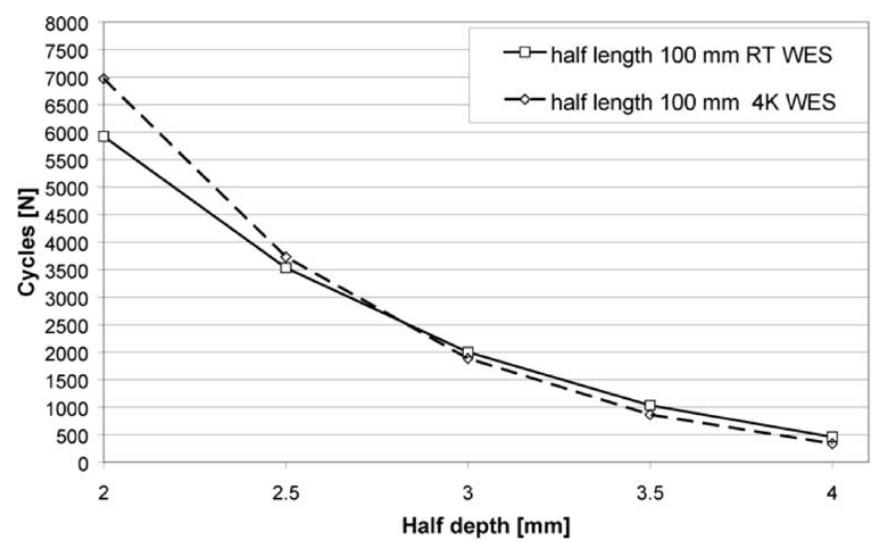

Fig. 10. Fatigue cycles for an embedded crack; WES comparison RT \& 4 K.

This correction factor increases the safety margin providing a very conservative estimation of $\delta_{c}$ equivalent to a much lower value of $K_{I c}$ with respect to the assumed $100 \mathrm{MPa} \sqrt{ } \mathrm{m}$.

For the dipole longitudinal weld in design conditions, $\delta_{c}$ is between 0.040 and $0.060 \mathrm{~mm}$.

For a surface crack the results are shown in Fig. 5.

For an embedded crack the results are shown in Fig. 6.

For both cases there is an asymptote: with a total depth of $\sim 2.4 \mathrm{~mm}$ for surface flaws and a half depth of $\sim 2.8 \mathrm{~mm}$ for embedded flaws. Below these values of $a$, even for the cases with $m=1.6$ and the maximum tensile stress, there are no 
reasonable values of flaw length for which the defect becomes critical.

To reduce the risk due to the presence of more than one defect a coupling law to define the minimum distance between two coplanar flaws is under study [3].

\section{CONCLUSION}

As the results of this work the LHC main dipole design shows very high level of reliability respect to possible flaws on the longitudinal welds and their possible propagation. The calculation demonstrates that an embedded flaw with total depth up to $5.6 \mathrm{~mm}$ or a surface flaw with total depth up to $2.4 \mathrm{~mm}$ do not represent a possible origin of the risk.

The fatigue cycles have negligible effect on critical dimensions.

\section{APPENDIX}

In this part of the paper the results of others methods and approaches used for this study have been summarized.

\section{A. Leak Before Break According to Barsom-Rolfe Approach [5]}

In order to estimate the necessary toughness of the weld so that a crack could grow through the wall and the vessel would leak before fracturing the leak-before-break criterion can be used. If this criterion is satisfied, the critical size at the design stress level would be greater than the thickness.

The application of this method shows, for the maximum acceptable value of stress, a safety margin of a factor two-three respect to the design value.

\section{B. Fatigue Crack Growth}

Because for the longitudinal weld the design number of cycles is 25 an improved formula [8] based on the LCF (Low Cycle Fatigue) properties has been used to better describe the fatigue crack growth for low number of cycle. This formula has a lower limit of validity at around 100 cycles and improves the Paris Law being the prediction made from the LCF properties, the fatigue threshold $\Delta K_{t h}$ and the local mean stress.

$$
\frac{d a}{d N}=\frac{\Delta K^{2}-\Delta K_{t h}^{2}}{\frac{1}{2} \pi E\left(\sigma_{y}^{\prime}\right)^{1-\frac{1}{\beta}}\left[4 e_{f}^{\prime}\left(1+n^{\prime}\right)\left(\sigma_{f}^{\prime}-\sigma_{m}^{*}\right)\right]^{\frac{1}{\beta}}}
$$

$\sigma_{y}^{\prime} \quad$ cyclic yield strength

$\beta \quad-(b+c)$

$b \quad$ fatigue strength exponent

$c \quad$ fatigue ductility exponent

$\varepsilon_{f}^{\prime} \quad$ fatigue ductility coefficient

$n^{\prime} \quad$ cyclic strain hardening exponent

$\sigma_{f}^{\prime} \quad$ fatigue strength coefficient

$\sigma_{m}^{*} \quad$ local mean stress

$\Delta \mathrm{K}$ variation of the stress intensity factor with the cycles

Due to the lack of information for the AISI $316 \mathrm{LN}$, the data available for the AISI 304 at room temperature have been used as the first approximation, knowing that the $316 \mathrm{LN}$ should have at least the same performances or even better.

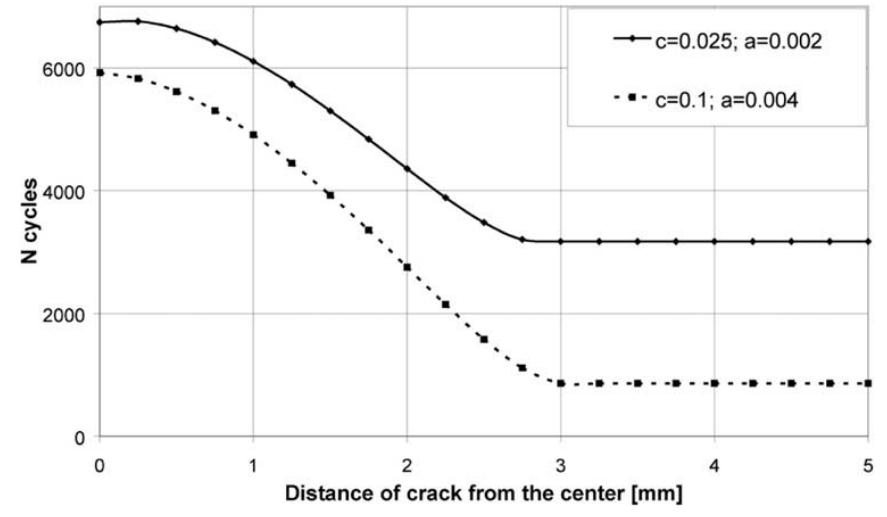

Fig. 11. Embedded eccentric crack: fatigue cycles, where $c$ is the half length and $a$ is the half depth.

We also compare the results with the law formula using the data for $m$ and $C$ proposed in the Barson and Rolfe [5].

Making a comparison of all the approaches, the most conservative one is the WES approach at $4 \mathrm{~K}$ for an embedded crack, as it is possible to see in the previous figures.

From those graphics it is possible to confirm that, for the number of cycles of the dipole, a crack cannot become critical due to the fatigue.

Moreover, considering an embedded eccentric crack it is possible to determine the fatigue life in function of the eccentricity. Fig. 11 shows that the minimum life in presence of an eccentric embedded crack is determined when it can be considered as a surface crack; it means when the extremity of the flaw catches up the surface.

\section{ACKNOWLEDGMENT}

The authors thank the CERN library and the Interlibrary Loan Service for the precious and efficient collaboration and help. Many thanks as well to professor C. S. Lee (Dept. of Materials Science \& Engineering Pohang Univ. of Science and Technology, Korea) for his communications.

\section{REFERENCES}

[1] S. Kane, Fracture Toughness Requirements for RHIC Cryogenic Design: Brookhaven National Laboratory, 1992.

[2] J. Kerby and D. Chichili, MQXB Longitudinal Weld Analysis, Fermilab, $17 / 02 / 2000$.

[3] Method of Assessment for Flaws in Fusion Welded Joints With Respect to Brittle Fracture and Fatigue Crack Growth, WES 2805/1997 Japan Welding Engineering Society Standard, .

[4] S. Hayashi, "Large Barrel Cryostat Repair: Assessment of the Chimney Weld Joint," unpublished.

[5] J. M. Barsom and S. T. Rolfe, Fracture and Fatigue Control in Structures: Prentice Hall.

[6] R. L. Tobler et al., "Austenitic stainless steel welds," Cryogenics, vol. 26, July 1986.

[7] M. Bajko, P. Fessia, and D. Perini, "Statistical studies of the robustness of the LHC main dipole mechanical structure," IEEE Trans. Appl. Supercond., vol. 10, no. 1, pp. 77-80, March 2000.

[8] J. C. Newman Jr. and I. S. Raju, "An empirical stress-intensity factor equation for the surface crack," Engineering Fracture Mechanics, vol. 15, no. 1-2, pp. 185-192, 1981, Pergamon Press Ltd..

[9] D. M. Li, W. J. Nam, and C. S. Lee, "An improvement on prediction of fatigue crack growth from low cycle fatigue properties," Engineering Fracture Mechanics, vol. 60, no. 4, pp. 397-406, 1998, Pergamon Press Ltd.. 